\title{
Flow cytometric DNA analysis in longstanding ulcerative colitis: a method of prediction of dysplasia and carcinoma development?
}

\author{
R LÖFBERG, B TRIBUKAIT, $\AA$ ÖST, O BROSTRÖM, AND H REICHARD \\ From the Unit of Gastroenterology, Department of Medicine, Huddinge University Hospital, Department of \\ Medical Radiobiology, Karolinska Institutet, Department of Pathology and Medical Department II, South \\ Hospital, Stockholm, Sweden
}

SUMmARY During a prospective study lasting 3.5 years flow cytometric DNA analysis was evaluated as a possible predictor of dysplastic and malignant lesions in longstanding ulcerative colitis. Fifty three patients with total ulcerative colitis (mean disease duration of 22 years) were regularly colonoscoped. Biopsies of colonic mucosa were analysed by flow cytometric technique and were also assessed histologically. Findings of abnormal DNA pattern (aneuploidy) were compared with findings of dysplasia. Five patients $(9 \%)$ had aneuploidy, four of those at repeated colonoscopies. Four of those patients also had various degrees of dysplasia. In one patient aneuploidy preceded the finding of dysplasia and in another aneuploidy preceded a well differentiated adenocarcinoma, grade Dukes' A subsequently found at surgery. Four additional patients had dysplasia, all in connection with macroscopic lesions, but were diploid. It is suggested that flow cytometric DNA analysis in long standing ulcerative colitis may be helpful in addition to histopathology in the detection of potential malignancy in ulcerative colitis.

The cancer risk in ulcerative colitis is one of the major clinical problems in the long term management of the disease..$^{1-3}$ The current concept of surveillance by annual colonoscopies with multiple biopsies, is based on detection of dysplasia in selecting patients for prophylactic colectomy. ${ }^{45}$

Histological grading of dysplasia requires an experienced pathologist, adherence to a strict classification, ${ }^{6}$ and must be separated from reactive inflammatory changes in the epithelium. When dysplasia is detected, there is still a risk that carcinoma may already be present. ' An additional and possibly more objective method for earlier detection of potential malignancy is therefore desirable.

Studies using rapid flow cytometric technique for DNA analysis of numerous cells in biopsies or surgical specimens from various malignancies, have

Address for correspondence: Dr Robert löfberg. Unit of Gastroenterology, Department of Medicine. Huddinge University Hospital, S-141 86 Huddinge. Sweden.

Received for publication 5 February 1987. in many cases shown an abnormal, aneuploid DNA pattern indicating gross chromosomal changes. ${ }^{\mathrm{x}}$ In idiopathic colonic carcinoma aneuploidy has been demonstrated in about $85 \%$ of the cases. ${ }^{9}$ DNA analyses of cell material from patients with ulcerative colitis have recently shown aneuploid DNA patterns in about $10 \%$ of patients," and in one case a subsequent development of carcinoma has been reported."

In this report the results from a prospective study during 3.5 years are reported. The aim of the study was to investigate whether presence of aneuploidy in patients with longstanding ulcerative colitis is related to development of dysplasia or carcinoma. Furthermore the distribution and the persistency of aneuploidy at repeated examinations with colonoscopy was evaluated.

\section{Methods}

PATIENTS

Between 1982 and 198553 patients with ulcerative 
colitis of 10 years duration were examined with regular colonoscopies, as part of an already existing surveillance programme based on dysplasia development. ${ }^{5}$ The mean duration of disease was $22 \cdot 0$ years with a median of 20 years (range 10-43).

At colonoscopy two biopsies were taken from 10 locations in the colon (Fig. 1). Further biopsies were taken if macroscopic polypoid or raised granular lesions were found. One biopsy from each location was assessed histologically, and one taken immediately adjacent $(0-2 \mathrm{~mm}$ apart) was analysed for DNA content by flow cytometric technique described previously ${ }^{12}$ and summarised below. The two biopsies were taken as close together as possible, to ensure comparison between findings of dysplastic and/or aneuploidy in the same part of the mucosa. Dysplasia was graded by one pathologist according to the new classification adopted by Riddell $e$ et al" as negative; indefinite, probably negative; indefinite, probably positive; low grade or high grade. Dysplasia was only recorded if the mucosa showed no or only slight inflammation - that is, only inflammatory round cells in the stroma - to avoid misinterpretation of reactive dysplasia caused by inflammation.

The biopsies for DNA-analysis were pooled in three different fractions: (a) caecum, ascending colon and hepatic flexure (locations $1,2,3$ ); (b) transverse colon and splenic flexure (locations $4,5,6$ ); (c) descending, sigmoid colon and rectum (locations 7, 8, 9, 10) (Fig. 1).

If aneuploidy was detected, however, biopsy specimens from subsequent colonoscopies were, if feas-

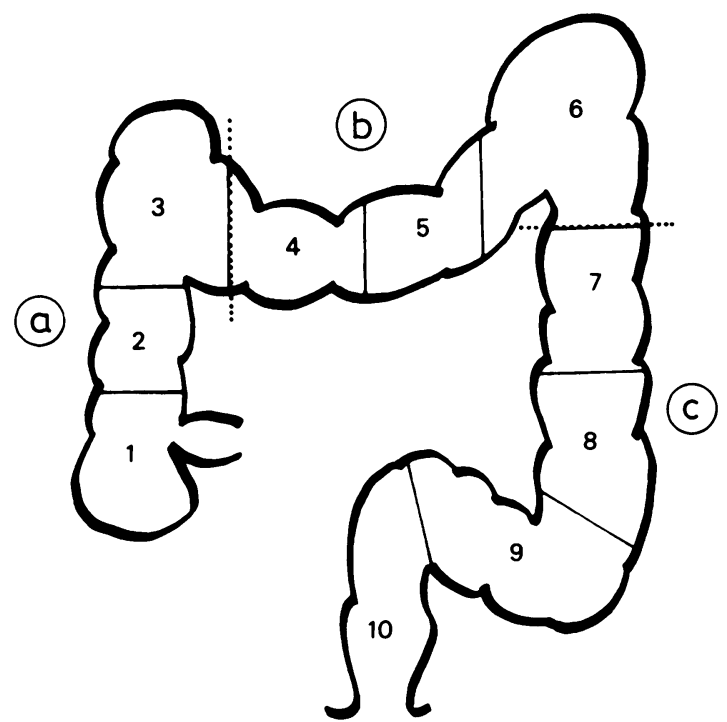

Fig. 1 Biopsies were sampled from 10 locations in the colon (1-10). For DNA analysis biopsies were pooled into three groups $(a-c)$. ible, analysed from all 10 locations separately. If aneuploidy and/or dysplasia was found, the examination was repeated within one year.

\section{FLOW CYTOMETRIC DNA ANALYSIS}

The cell material for DNA analysis was placed in saline, pressed through a nylon grid (40-50 mesh counts $/ \mathrm{cm}$ ) and the resulting suspension fixed in ice cold $96 \%$ ethanol. The fixed cells were then treated with a isotonic Tris EDTA buffer (Tris $0 \cdot 1 \mathrm{M}, \mathrm{NaCl}$ $0.07 \mathrm{M}$ and EDTA $0.005 \mathrm{M}, \mathrm{pH} \mathrm{7.5)}$ containing RNAse $(1 \mathrm{mg} / \mathrm{ml})$ in order to eliminate the RNA. Suspensions of single cell nuclei were obtained by pepsin treatment $(0.5 \%$ pepsin solution, $\mathrm{pH} 2 \cdot())$ for 10 minutes in a $37^{\circ} \mathrm{C}$ water bath. This was followed by staining of the nuclei with $2.5 \times 10^{5} \mathrm{M}$ ethidium bromide in Tris EDTA buffer with a molarity of 395 mOsm. This high molarity further reduced the risk of non-specific binding of ethidium bromide.

The DNA content of the cell nuclei was analysed by using a rapid flow cytofluorometer ICP 11 (Phywe, W Germany), equipped with a xenon lamp. The excitation and emission wave lengths were 455-490 $\mathrm{nm}$ and $590-630 \mathrm{~nm}$ respectively. The output was sorted with a 256 multichannel analyser.

The DNA values of the analysed cells were calculated in relation to the DNA content of normal human lymphocytes, with a coefficient of variation below $3 \%$ in order to get a normal diploid reference point. The ploidy level of cell populations was defined as the DNA value of the $G_{1} / G_{0}$ cells in relation to the ploidy level of normal diploid cells. If the ploidy level deviated less than $10 \%$ from the standard lymphocytes it was regarded as diploid (Fig. 2 ). In this case the majority of cells are located in the first peak at 2 c corresponding to 46 normal chromosomes. A small fraction (around 5\% of the cells) are located in the second, tetraploid peak at $4 \mathrm{c}\left(\mathrm{G}_{2}+\mathrm{M}\right)$

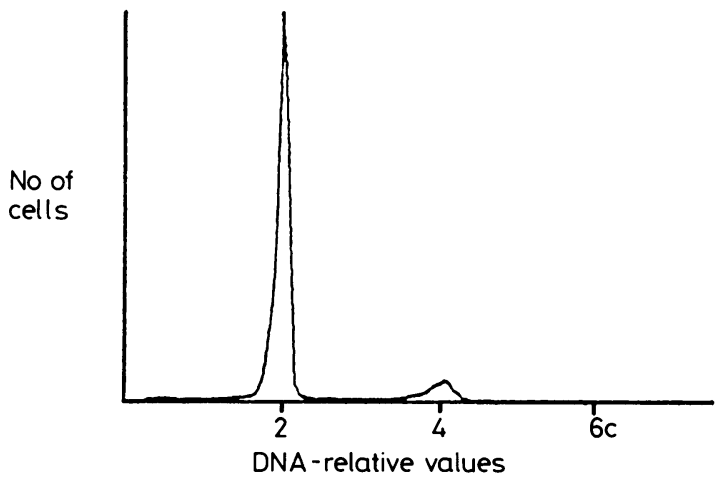

Fig. 2 Normal DNA histogram of colonic mucosa in ulcerative colitis. The peak at 2 c represents normal diploid $G_{1}$ cells. To the right at $4 c G_{2}+M$ cells are seen. Between these two peaks is the proliferative $S$-phase. 
with the double number of chromosomes. In between those peaks are the cells in the proliferative S-phase. Thus, $\mathrm{G}_{1}$-peaks with c-values exceeding $2 \cdot 2 \mathrm{c}$ were considered as aneuploid. The higher the c-values, the higher the total amount of chromosomes. The number of cells analysed from each preparation varied from a few thousand to 50000 with an average of about 25000 .

The proportions of cells in the various parts of the cell cycle were calculated using a simplified method described by Baisch et al. ${ }^{13}$ It was assumed that the $\mathrm{G}_{1}$ and $\mathrm{G}_{2}+\mathrm{M}$ peaks had Gaussian distributions and that the S-phase was represented by a rectangular area between the channel numbers were the $G_{1}$ and

Table 1 Results of flow cytometric DNA-analyses and histological findings of dysplasia in 53 patients with longstanding total ulcerative colitis

\begin{tabular}{llll}
\hline & Dysplasia & & \\
\cline { 2 - 4 } & & $\begin{array}{l}\text { Indefinite, } \\
\text { probably } \\
\text { negative }\end{array}$ & $\begin{array}{l}\text { Indefinite, } \\
\text { probably positive } \\
\text { andloworhigh } \\
\text { grade }\end{array}$ \\
DNA-analyses & Negative & 4 & $4^{*}$ \\
\hline Diploid & 40 & 0 & 4 \\
Ancuploid & 1 & 0 & \\
\hline
\end{tabular}

${ }^{*}$ Dysplasia in macroscopic polypoid or nodular lesions only.
$\mathrm{G}_{2}+\mathrm{M}$ peaks had their maximum values. Cell numbers were calculated from the multichannel analyser by automatic integration after subtraction of the background.

\section{Results}

A total of 85 colonoscopies were carried out averaging 1.6 per patient (range 1-5). The frequency of findings of dysplasia and/or of aneuploidy among the patients is shown in Table 1. Five of the 53 patients $(9 \%)$ were shown to have aneuploid DNA patterns and those patients are described here and in Table 2. Mean duration of disease for those five patients was $25 \cdot 4$ years with a median of 23 years (range $15-35$ ).

\section{PATIENT 1}

At colonoscopy 1982 no dysplasia was found but the c-fraction of the DNA analysis showed aneuploidy $(3.6 \mathrm{c})$. Slightly more than a year later there were neither signs of dysplasia nor DNA abnormalities, but the cell material for the DNA analysis was sparse. In 1985 the 10 different biopsy specimens were analysed separately by flow cytometry and in the sigmoid colon (location 9) aneuploidy $(3.7 \mathrm{c}$ ) was once again found, corresponding to the aneuploidy in fraction c 1982. No dysplasia was found in this location or in any other part of the colon (Fig. 3).

Table 2 Individual results of dysplasia and aneuploid DNA-patterns in five patients at consecutive colonoscopies. Fractions and locations of biopsies are according to Fig. I

\begin{tabular}{|c|c|c|c|c|c|c|c|}
\hline \multirow[b]{2}{*}{ Patient (n) } & \multirow[b]{2}{*}{ Age (yrs) } & \multirow{2}{*}{$\begin{array}{l}\text { Disease duration } \\
\text { (yrs) }\end{array}$} & \multirow{2}{*}{$\begin{array}{l}\text { Colonoscopy } \\
\text { year }\end{array}$} & \multicolumn{2}{|c|}{ DNA-analysis } & \multicolumn{2}{|l|}{ Histology } \\
\hline & & & & aneuploidy & fract/loc & dysplasia & location \\
\hline \multirow[t]{3}{*}{1} & \multirow[t]{3}{*}{63} & \multirow[t]{3}{*}{35} & 1982 & + & $\mathrm{C}$ & - & \\
\hline & & & 1984 & - & & - & \\
\hline & & & 1985 & + & 9 & - & \\
\hline \multirow[t]{8}{*}{2} & \multirow[t]{8}{*}{30} & \multirow[t]{8}{*}{19} & 1982 & + & B. C & - & \\
\hline & & & 1983 & + & A. B & ++ & 1 \\
\hline & & & 1984 & + & $\mathrm{C}$ & + & 3,4 \\
\hline & & & & & & ++ & 7 \\
\hline & & & 1985 & + & A. B. C & + & 3 \\
\hline & & & & & & ++ & 1 \\
\hline & & & 1985 & + & $4,7,8,10$ & + & 9 \\
\hline & & & & & & ++ & 5 \\
\hline \multirow[t]{4}{*}{3} & \multirow[t]{4}{*}{45} & \multirow[t]{4}{*}{23} & 1983 & + & A, B & + & $3-6,8$ \\
\hline & & & & & & ++ & 7.9 \\
\hline & & & 1984 & + & B & + & $6,8,9$ \\
\hline & & & 1985 & + & A, B, C & + & 3,8 \\
\hline \multirow[t]{6}{*}{4} & \multirow[t]{6}{*}{32} & \multirow[t]{6}{*}{16} & 1984 & + & A, B & + & $6,9^{*}$ \\
\hline & & & & & & ++ & $2,3^{*}$ \\
\hline & & & 1985 & + & $2,3,6,7$ & + & 7 \\
\hline & & & (colectomy & & 8.10 & ++ & $3,4,8$ \\
\hline & & & specimen) & & & +++ & 2 \\
\hline & & & & & & Ca Dukes' A & 2 \\
\hline 5 & 45 & 35 & 1985 & + & B & + & 7,8 \\
\hline
\end{tabular}

Aneuploidy: + present, - not present. Dysplasia: - negative, + probably positive, ++ low grade. +++ high grade. ${ }^{*}$ additional macroscopic lesions with dysplasia. 


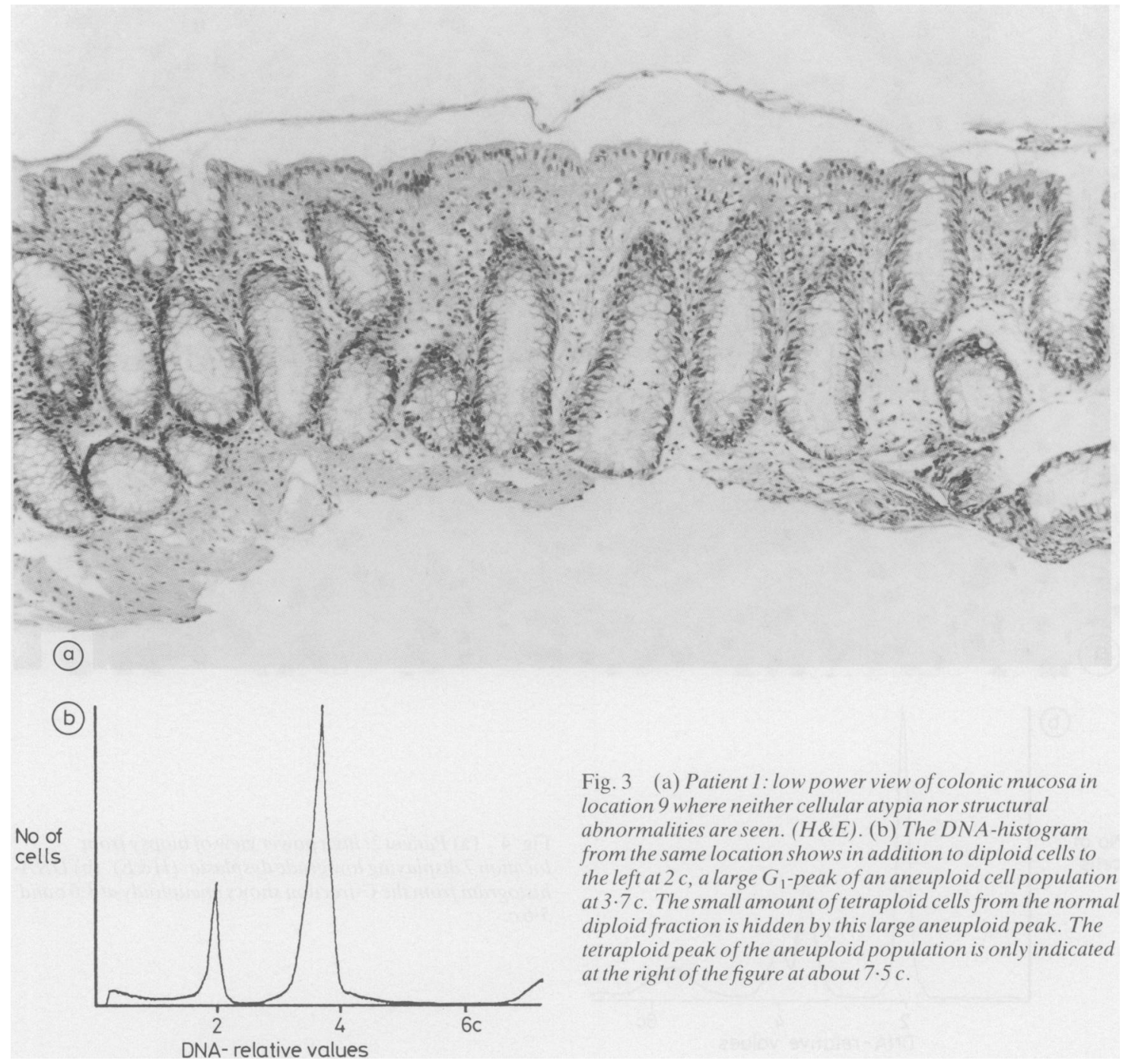

PATIENT 2

Aneuploidy in fractions b (3.8c) and c (3.6 c, 4.6 c) was found at the colonoscopy in 1982 but no dysplasia was detected. One year later aneuploidy was found in fraction a $(4.0 \mathrm{c})$ and $\mathrm{b}(4.6 \mathrm{c})$, and low grade dysplasia was detected in flat mucosa in the caecum (location 1). In 1984 fraction c showed aneuploidy $(3.6 \mathrm{c}, 5.6 \mathrm{c})$, while probable positive dysplasia was found in locations 3 and 4 and low grade dysplasia in location 7 (Fig. 4). Repeated colonoscopy in 1985 revealed aneuploidy in fraction a $(3.6 \mathrm{c}), \mathrm{b}(3.5 \mathrm{c})$, and $c(3.5 \mathrm{c})$ and again low grade dysplasia in the caecum. At the latest colonoscopy in 1985 the DNA analysis showed aneuploidy in locations $4(3.6 \mathrm{c}), 7$
$(2.3 \mathrm{c}), 8(4.7 \mathrm{c})$ and $10(3.6 \mathrm{c})$. Probably positive dysplasia was found in location 9 and low grade dysplasia in location 5 .

PATIENT 3

At the first colonoscopy in 1983 aneuploidy was present in fractions a $(3.4 \mathrm{c}), \mathrm{b}(3.6 \mathrm{c})$ and probably positive dysplasia in flat mucosa was present in locations 3-6 and 8, as well as low grade dysplasia in 7 and 9. The subsequent two colonoscopies revealed only probable dysplastic changes. In 1984 aneuploidy $(3.2 \mathrm{c})$ was found in fraction $\mathrm{b}$, and in 1985 in a $(3.6 \mathrm{c}), \mathrm{b}(3.1 \mathrm{c})$, and c (3.5 c) comprising $30-90 \%$ of all cells. 


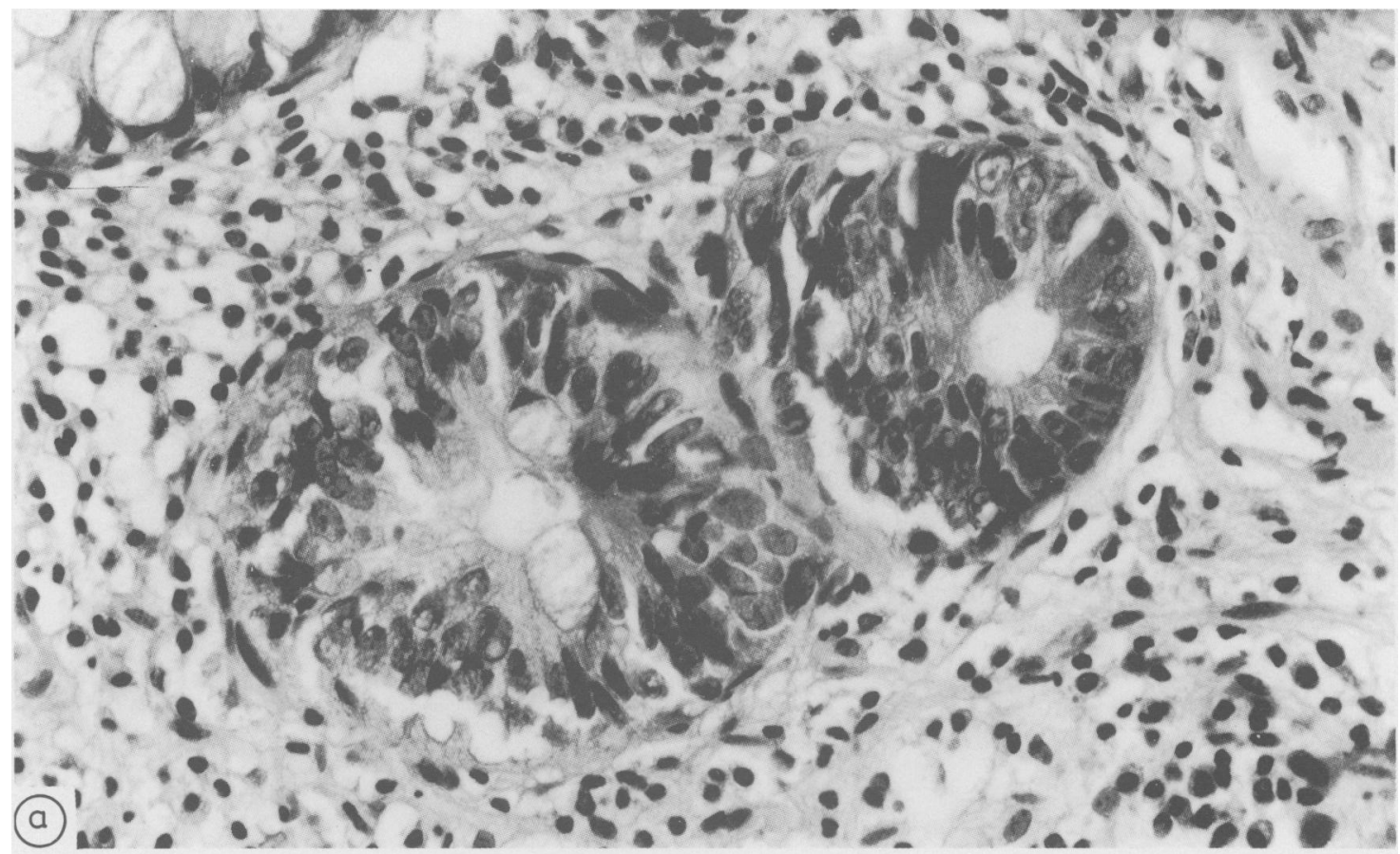

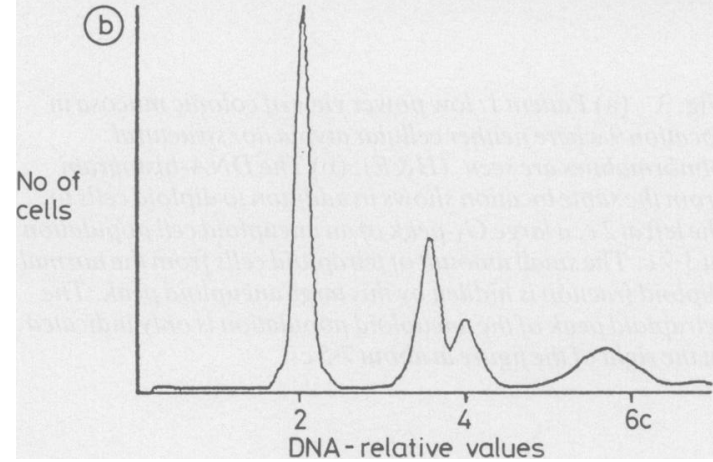

PATIENT 4

The colonoscopy in 1984 showed low grade dysplasia in location 2 and 3 and also macroscopic lesions with probably positive and low grade dysplasia. Fraction a $(3.3 \mathrm{c})$ and b $(3.4 \mathrm{c})$ of the DNA-analysis showed aneuploidy. Another colonoscopy shortly afterwards revealed a transformation into high grade dysplasia in the flat mucosa. Colectomy was done in the beginning of 1985 and a well differentiated adenocarcinoma (Dukes' A) of the ascending colon, corresponding to location 2 was found at the histopathological examination. High grade dysplasia was found in location 2 and low grade dysplasia in locations 3,4 , and 8 . Specimens from the resected
Fig. 4 (a) Patient 2: high power view of biopsy from location 7 displaying low grade dysplasia. ( $H \& E)$. (b) DNAhistogram from the C-fraction shows aneuploidy at $3 \cdot 6 \mathrm{c}$ and $5 \cdot 6 \mathrm{c}$.

colon taken immediately adjacent to the histological specimens showed aneuploidy in locations $2(4.0 \mathrm{c})$, $3(4 \cdot 0 \mathrm{c}), 6(4 \cdot 0 \mathrm{c}), 7(2 \cdot 9 \mathrm{c}), 8(3 \cdot 0 \mathrm{c})$ and $10(3 \cdot 0 \mathrm{c})$.

\section{PATIENT 5}

Aneuploidy was detected in fraction b $(3.6 \mathrm{c})$ at colonoscopy in 1985 . Probable positive dysplasia was found in locations 7 and 8 .

Four additional patients had dysplasia connected with macroscopic, polypoid lesions but none had aneuploidy. Two patients had lesions classified as tubular and tubulo-villous adenomas respectively, both with low grade dysplasia. One patient had a macroscopic lesion with probable positive dysplasia, 
which was completely removed by polypectomy. The fourth patient had a persistent macroscopic nodular lesion with low grade dysplasia and was referred to surgery, but no further dysplasia or cancer was revealed. Mean age of those four patients was 51 years (range 40-57) with a mean duration of disease of $28 \cdot 5$ years (range 13-41).

Eleven colonoscopies carried out in 10 other patients revealed moderate inflammation, histologically in some locations. Four of those patients had reactive changes in the epithelium, classified as indefinite for dysplasia, probably negative. None of those had aneuploidy.

The percentage of cells in the S-phase varied between $0 \cdot 3-20 \cdot 2$ when all evaluable DNA analyses were considered. In samples with aneuploid DNA pattern the mean percentage of cells in the S-phase was 9.5 (range $2 \cdot()-20 \cdot 2 \%$ ). In the four patients having macroscopic lesions with dysplasia but normal DNA pattern, the mean S-phase value was $5 \cdot 3 \%$ (range $2 \cdot()-8 \cdot 4 \%, \mathrm{SD} \pm 1 \cdot 7$ ). In samples without aneuploidy or dysplasia the mean S-phase value was $5 \cdot 7 \%$ (range $1 \cdot 2-17 \cdot 1 \%, \mathrm{SD} \pm 2 \cdot 6$ ).

\section{Discussion}

This is the first prospective study describing the development and persistency of aneuploidy in flow cytometric DNA analyses of colonic mucosal biopsies in a patient group with longstanding total ulcerative colitis. Special emphasis was made to correlate the findings of aneuploidy with histological dysplasia by taking the biopsies from each segment of the colon close to each other.

In five of 53 patients aneuploidy was detected. Four patients had aneuploidy at repeated occasions suggesting that once established the changes in DNA pattern may persist. Aneuploidy was sometimes detected multifocally and the sites where aneuploidy was found in individual patients also changed at subsequent colonoscopies. This might be explained by a patchy distribution of aneuploidy, and/or sampling error because of the difficulty in taking the biopsies in exactly the same place from one examination to another. Variations in ploidy level may be explained by the fact that multiple cell lines with differing degrees of aneuploidy have been demonstrated in patients with idiopathic colonic carcinoma. ${ }^{9}$

Four of the patients with aneuploidy also had different degrees of dysplasia in flat mucosa that were aneuploid although not always in the same locations. This can partly be explained by the fact that dysplasia has a patchy distribution in the colon, ${ }^{1+}$ but is nevertheless difficult to explain completely as the biopsies were taken close to each other.
Another small group of patients had dysplasia in connection with macroscopically detected polypoid lesions but no aneuploidy. Two had adenomas which may be associated with ulcerative colitis, but may also be age related. The significance of findings of adenoma for subsequent carcinoma development in ulcerative colitis is not clear. ' Determination of Sphase values in this small group showed no significant difference compared with the rest of the patients with neither dysplasia nor aneuploidy. Thus, the S-phase value did not contribute further information in this patient group.

Four other patients had reactive, inflammatory changes of the epithelium, considered as indefinite for dysplasia, probably negative at the histological examination. None of those patients had aneuploidy. This finding suggests an advantage of the method with flow cytometric DNA analysis as it may help to discriminate between reactive changes and true dysplasia. In the two latter groups of patients long term surveillance must be undertaken before any conclusions of the predictive value of the DNA pattern can be made.

Previous studies have shown that aneuploid DNA pattern is closely correlated with malignancy. ${ }^{\times}$There are no prospective studies, however, showing the value of aneuploidy as an early predictor of malignancy in known premalignant conditions such as atrophic gastritis or longstanding ulcerative colitis. Furthermore it is not known if aneuploidy is irreversible in these conditions. Experimental studies, in fact, have shown that when bronchial epithelium in dogs was treated with a carcinogen, cytological atypia and malignancy developed as well as severe aneuploidy, but in $66 \%$ of the dogs the changes were reversible when the carcinogenic treatment was stopped. Similar results were seen in cigarette smokers who stopped or decreased smoking. ${ }^{15}$ Although in ulcerative colitis the factor(s) inducing carcinoma development may work over a much longer period of time. Our results so far are not conclusive as to whether aneuploidy is a reversible condition when found in patients with longstanding total ulcerative colitis.

The two patients in our study where the findings of aneuploidy preceded low grade dysplasia and carcinoma Dukes' A respectively, may indicate that a finding of a pathological DNA pattern is a predictor of impending malignancy. This indication is enhanced by the case previously reported by Hammarberg et al."

In one patient aneuploidy appeared without concomitant dysplasia at two colonoscopies two years apart, and this raises the question of how early is the change in DNA pattern.

As the flow cytometric DNA analysis only reveals 
gross chromosomal changes, patients in our study may develop malignancy with only minor chromosomal abnormalities which are undetectable with the method used. From DNA analyses in patients with idiopathic colonic carcinoma, it has been demonstrated that approximately $15 \%$ of the tumours have a normal, diploid DNA pattern." Carcinoma may also develop without association of dysplasia and this has been the case in approximately $20 \%$ of the colon cancers in ulcerative colitis patients. ${ }^{14}$ Both histology and DNA analyses may therefore miss some patients who ultimately will get carcinoma of the colon, but with regular colonoscopies the risk of missing an incurable carcinoma appears to be low. ${ }^{5}$

In conclusion aneuploidy was found in a small number of patients $(9 \%)$ with total longstanding ulcerative colitis. Once established the changes in DNA pattern seemed to persist. Aneuploidy was detected multifocally in the colon and was correlated to findings of dysplasia in flat mucosa but not necessarily in the same segment. Flow cytometric DNA analyses in patients with longstanding ulcerative colitis may become a complement to conventional methods in trying to detect patients with an increased risk of developing colonic carcinoma.

This study was partly supported by funds from the Cancer Society of Stockholm.

\section{References}

1 Edwards FC, Truelove SC. Carcinoma of the colon. Gut 1964; 5: 15-22.

2 deDombal FT, Watts JM, Watkinson G, Goligher JC. Local complications of ulcerative colitis: stricture, pseudopolyposis and carcinoma of colon and rectum. Br Med J 1966; 1: 1442-47.
3 Kewenter J, Ahlman H, Hultén L. Cancer risk inextensive ulcerative colitis. Ann Surg 1978; 188: 824-8.

4 Lennard-Jones JE, Ritchie JK, Morson C, Williams CB. Cancer surveillance in ulcerative colitis. Experience over 15 years. Lancet 1983; ii: 149-52.

5 Broström O, Löfberg R, Öst $\AA$, Reichard H. Cancer surveillance of patients with longstanding ulcerative colitis - a clinical, endoscopical and histological study. Gut 1986; 27: 1408-13.

6 Riddell RH, Goldman H, Ransohoff D, et al. Dysplasia in inflammatory bowel disease: Standardized classification with provisional clinical applications. Hum Pathol 1983; 14: 931-66.

7 Riddell RH. Dysplasia and cancer in ulcerative colitis: a soluble problem. Scand J Gastroenterol 1984; 19 suppl 104: 137-49.

8 Tribukait B. Clinical DNA flow cytometry. Med Oncol Tumor Pharmacother 1984; 1: 211-8.

9 Tribukait B, Hammarberg C, Rubio C. Ploidy and proliferations patterns in colorectal adenocarcinomas related to Dukes' classification and to histopathological differentiation. Acta Path Microbiol Immun Scand Sect A 1983; 91: 89-95.

10 Hammarberg C, Slezak P, Tribukait B. Early detection of malignancy in ulcerative colitis. A flow cytometric DNA study. Cancer 1984; 53: 291-5.

11 Hammarberg C, Rubio C, Slezak P, Tribukait B, Öhman U. Flow cytometric DNA-analysis as a means for early detection of malignancy in patients with chronic ulcerative colitis. Gut 1984; 25 : 905-8.

12 Tribukait B. Flow cytometry in surgical pathology and cytology of tumors of the genito-urinary tract. Adv Clin Cytol 1984; 2: 163-89.

13 Baisch H, Göhde W, Linden W. Analysis of PCP-data to determine the fraction of cells in the various phases of cell cycle. Rad Environ Biophys 1975; 12: 31-9.

14 Riddell RH. The precarcinomatous phase of ulcerative colitis. Curr Top Pathol 1976; 63: 179-219.

15 Auer G, Ono J, Nasiell M, Caspersson T, et al. Reversibility of bronchial cell atypia. Cancer Res 1982; 42: 4241-7. 